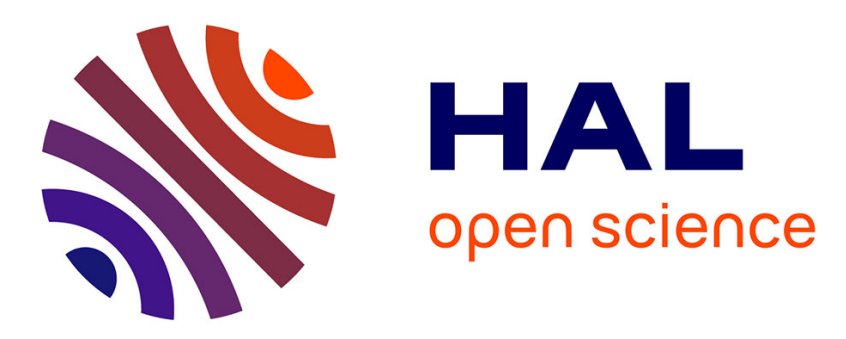

\title{
Protein arginine methylation in estrogen signaling and estrogen-related cancers.
}

Catherine Teyssier, Muriel Le Romancer, Stéphanie Sentis, Stéphan Jalaguier, Laura Corbo, Vincent Vc Cavaillès

\section{To cite this version:}

Catherine Teyssier, Muriel Le Romancer, Stéphanie Sentis, Stéphan Jalaguier, Laura Corbo, et al.. Protein arginine methylation in estrogen signaling and estrogen-related cancers.. Trends in Endocrinology and Metabolism = Trends in Endocrinology \& Metabolism , 2010, 21 (3), pp.181-9. 10.1016/j.tem.2009.11.002 . inserm-00452350

\section{HAL Id: inserm-00452350 https://www.hal.inserm.fr/inserm-00452350}

Submitted on 2 Feb 2010

HAL is a multi-disciplinary open access archive for the deposit and dissemination of scientific research documents, whether they are published or not. The documents may come from teaching and research institutions in France or abroad, or from public or private research centers.
L'archive ouverte pluridisciplinaire HAL, est destinée au dépôt et à la diffusion de documents scientifiques de niveau recherche, publiés ou non, émanant des établissements d'enseignement et de recherche français ou étrangers, des laboratoires publics ou privés. 


\section{REVIEW ARTICLE}

2

3

4

5

6

7

8

9

10

11 Catherine Teyssier ${ }^{1}$, Muriel Le Romancer ${ }^{2}$, Stéphanie Sentis ${ }^{2}$, Stéphan Jalaguier ${ }^{3}$, Laura 12 Corbo $^{2}$ and Vincent Cavaillès ${ }^{3}$

${ }^{1}$ INSERM, U554, Montpellier, F-34090, France ; CNRS, UMR5048, Centre de Biochimie

17 Structurale, Universités Montpellier 1 \& 2, Montpellier, F-34090, France

$18{ }^{2}$ Université de Lyon 1, ISPB and IFR62, Lyon F-69003, France; Equipe labellisée "La

19 Ligue,” ; U590 INSERM, Centre Léon Bérard, 28 rue Laennec, Lyon, F-69003, France

$20{ }^{3}$ IRCM, Institut de Recherche en Cancérologie de Montpellier, Montpellier, F-34298, France;

21 INSERM, U896, Montpellier, F-34298, France ; Université Montpellier1, Montpellier, F-

22 34298, France ; CRLC Val d'Aurelle Paul Lamarque, Montpellier, F-34298, France

23

24 Corresponding author: Dr Vincent Cavaillès (v.cavailles@ valdorel.fnclcc.fr) 
Abstract:

28 Estrogen signaling pathways regulate cellular processes such as proliferation and 29 differentiation, and if deregulated, are involved in several human pathologies. Post30 translational modifications (PTMs) play important roles in estrogen signaling pathways. This 31 review focuses on recent findings pertinent to arginine methylation of non-histone proteins 32 and their implications in estrogen signaling. We describe protein arginine methyltransferases 33 and demethylases, the role of methylarginine proteins in estrogen action and cross-talk with 34 other PTMs such as phosphorylation and lysine methylation. The relationships between 35 various PTMs form a specific code which might play an important role in hormone signaling. 36 In addition, deregulations of arginine methylation or of enzymes responsible for these 37 modifications could be key events in estrogen-dependent cancers such as breast cancer. 
Arginine methylation was first described as a post-translational modification (PTM) of

histones in the late 1960s. However, only recently the responsible enzymes (Box 1) and wide variety of substrates of this modification have been identified. While the addition of each methyl group does not modify the charge of the residue, it does increase its bulkiness and hydrophobicity. Interactions of a methylated protein with its binding partners can therefore be affected by this modification and impact the physiological functions of the substrate protein. Methylarginine substrates include transcription factors, nucleic acid-binding factors, signal transducers, splicing factors and histones. Because of the large number of substrates, protein arginine methyltransferases (PRMTs) and arginine methylation regulate various cellular processes such as cell differentiation, DNA repair, RNA processing, signal transduction, cellular localization, and apoptosis [1-3]. In this review, we describe the recent studies implicating arginine methylation in estrogen transcriptional regulation and likewise, in estrogen-related diseases.

\section{Role of PRMTs and methylarginine proteins in estrogen signaling} Estrogen action - Genomic and non-genomic effects

Nuclear estrogen receptors act mainly as ligand-activated transcription factors [4]. The binding of estrogens such as $17 \beta$-estradiol (E2) induces a protein conformation change in the receptor that allows recruitment of coactivator complexes with chromatin-remodeling or histone-modifying activities [5] (Box 2). Steroid-regulated promoters recruit the ligand-bound receptors and regulatory proteins in an ordered, cyclical manner with multiple rounds of coactivator assembly and disassembly [6]. Histone acetylases and some methyltransferases lead to a more open chromatin and increase gene transcription. P300, an acetyltransferase, and PRMT1 and CARM1, two well-characterized PRMTs, cooperate synergistically to regulate 
hormone target genes [7]. The activated estrogen receptor can also bind corepressors, such as RIP140 [8], which recruit enzymes with histone deacetylase activity (HDAC) to repress transcription. Anti-estrogen compounds like tamoxifen prevent steroid action by inducing a conformation in the ligand binding pocket of the receptor that fails to bind coactivators and allows the recruitment of corepressor proteins (such as NCoR and SMRT) together with HDACs [9].

In addition to transcriptional regulation, $\mathrm{ER} \alpha$ also mediates events through its association with signaling molecules outside the nuclei and independent of its direct influence on the genome [10]. For example, estradiol triggers cell proliferation and cell survival through activation of MAPK kinases and Akt pathways [11]. These non-genomic actions of estrogens occur rapidly and independently of protein synthesis. At the molecular level, ER $\alpha$ palmitoylation anchors a pool of ER $\alpha$ at the plasma membrane [12] where it interacts with Src, PI3K and other scaffold proteins as MNAR (modulator of non-genomic activity of ER). This complex can therefore activate the downstream pathways [13]. Recently, novel non-genomic action of estrogens in breast cancer cells has been described, involving the association of membrane ER $\alpha$ with HDAC6. This association induces tubulin deacetylation, potentially contributing to estrogeninduced cell migration [14].

81

\section{Methylarginine proteins involved in estrogen action}

Arginine methylation affects estrogen-mediated transcription by modifying both histone and non-histone proteins. Since histone methylation has been widely described in a various number of reviews [15-17], this section focuses on methylation of non-histone proteins and their role in estrogen action (Table 1 and Figure 1).

\section{Estrogen receptor}


Because PRMT1 [18] and CARM1 (PRMT4) [19] are ER $\alpha$ coregulators (Box 2), ER $\alpha$ could be a target for arginine methylation. Concordant with this, a recent study described ER $\alpha$ as a methylarginine substrate [20]. In this study, Le Romancer et al. used in vitro methylation assays and showed that PRMT1, but not CARM1, methylated ER $\alpha$ within the DNA binding domain. Mutation of arginine 260 into alanine (R260A) specifically abolished the modification by PRMT1. An antibody specific to methylated R260 confirmed ER $\alpha$ methylation in living cells. Perhaps more interestingly, estradiol treatments of MCF7 cells drastically increased $\mathrm{ER} \alpha$ methylation within 5 minutes of treatment. A decrease of the methylated form was observed within less than one hour, suggesting enzymatic removal of the methyl group. Indeed, this disappearance was not due to ER $\alpha$ degradation by the proteasome. Moreover, immunohistochemical experiments performed on human breast tumors showed that the methylated form of ER $\alpha$ was exclusively localized in the cytoplasm of breast epithelial cells. Since rapid effects have been described for non-genomic estrogen actions, the role of methylated $\mathrm{ER} \alpha$ in those pathways was investigated. Interestingly, methylated ER $\alpha$ was essential for E2-induced assembly of ER $\alpha$ with Src, the p85 subunit of PI3K and the focal adhesion kinase (FAK), a Src substrate involved in the migration process.

E2 activation of Akt was not observed if ER $\alpha$ R260 was mutated to an alanine. Collectively, these results show that ER $\alpha$ methylation is a prerequisite for its association with certain molecules involved in growth factor signaling. Since formation of the ER $\alpha / \mathrm{Src} / \mathrm{PI} 3 \mathrm{~K} / \mathrm{FAK}$ complex activates Akt and corresponding downstream pathways, ER $\alpha$ methylation is likely involved in regulating cell proliferation and cell survival.

\section{Transcriptional coregulators}

Transcriptional coregulators play critical roles in controlling ER-mediated transcription. They function through protein-protein interactions, by facilitating or inhibiting recruitment of other coregulators or specific components of the transcription machinery (Box 2). Methylation of 
113 these cofactors may influence complex formation, enzymatic activity, subcellular localization,

114 and stability, leading to a subtle regulation of ER-mediated transcription (Figure 1).

115 SRC-3: $\mathrm{p} / \mathrm{CIP} / \mathrm{AIB} 1 / \mathrm{SRC}-3$ is a member of the $\mathrm{p} 160$ coactivator family involved in CARM1 116 and PRMT1 recruitment to ER target genes [7]. SRC-3 (steroid receptor coactivator-3)

117 protein levels are amplified in breast cancer and associated with poor prognosis [21, 22]. In

118 fact, studies classified SRC-3 as an authentic oncogene [23, 24]. Two independent groups

119 found that CARM1 modifies SRC-3 [25, 26], methylating it at the C-terminal region which

120 contains the p300 and CARM1-binding sites. SRC-3 methylation, which is induced by

121 estradiol, leads to the dissociation of CBP and CARM1 from SRC-3. Moreover, SRC-3

122 arginine methylation reduces its stability and causes an increase in its turnover. These results

123 show that arginine methylation is implicated in the regulation of coregulator stability in

124 response to estradiol. In addition to this role, arginine methylation also regulates the balance

125 between coactivator complex assembly and disassembly. Studies suggest that repetitive

126 association and dissociation of steroid receptors and coactivators from their target promoters

127 may be required to maintain an activated state of transcription [6]. Altogether, these results

128 highlight coactivator methylation as an important regulatory mechanism in hormonal

129 signaling.

130 CBP/p300: Recently, Lee et al. reported that p300 is methylated by CARM1 at R2142 which

131 is located within the C-terminal GRIP1 binding domain. Interestingly, methylation of R2142

132 inhibits the interaction between p300 and GRIP1 whereas PADI 4 removes this methylation

133 mark, thereby enhancing the p300-GRIP1 interaction. These methylation and demethylation

134 events alter the conformation and activity of the coactivator complex and regulate estrogen

135 receptor-mediated transcription [27]. This provides another example of arginine methylation

136 regulating coactivator complex assembly, conformation and function. 
137 PGC-1 $\alpha$ : PGC-1 $\alpha$ (peroxisome proliferator-activated receptor gamma coactivator 1 alpha)

138 serves as a coactivator for several nuclear receptors, including ER $\alpha$, as well as other 139 transcription factors, such as nuclear respiratory factor 1 (NRF-1). Expression of PGC-1 $\alpha$ is 140 induced by a variety of physiological stimuli that regulate metabolic activity, such as 141 exposure to cold, exercise, and fasting. PGC- $1 \alpha$ regulates metabolic processes by affecting 142 genes involved in mitochondrial biogenesis, respiration and gluconeogenesis. Teyssier et al. 143 showed that the C-terminal region of PGC-1 $\alpha$ is methylated by PRMT1 at one or more sites 144 within a glutamate and arginine rich region. PGC-1 $\alpha$ coactivator activity and the ability of 145 PGC-1 $\alpha$ to induce expression of target genes are both compromised by mutation of modified 146 arginine residues of PGC-1 $\alpha$ or as a result of reduced PRMT1 levels by siRNA. Because 147 inhibition of PRMT1 leads to inhibition of the expression of some PGC-1 $\alpha$ target genes 148 involved in mitochondrial biogenesis, it is tempting to speculate a role for PRMT1 in this 149 regulatory pathway [28]. However, the binding partners of methylated PGC-1 $\alpha$ remain to be 150 determined.

151 RIP140: RIP140 (Receptor Interacting Protein of $140 \mathrm{kDa}$ ) is a well-known ER hormone152 dependent binding corepressor [8]. In vitro and in vivo arginine methylation of RIP140 by 153 PRMT1 has been described in 3T3-L1 adipocytes [29], and liquid chromatography-tandem 154 mass spectroscopy identified that arginine methylation occurs on R240, R650, and R948 as a 155 mono-methyl mark, suppressing RIP140 repressive activity by two mechanisms. First, 156 methylation of R240, located in the HDAC3 interaction domain of RIP140, impairs its 157 interaction with HDAC3, reducing its repressive function. Second, arginine methylation of the 158 three sites increases RIP140's interaction with CRM1, a component of the export machinery; 159 this leads to RIP140 export to the cytoplasm, thereby reducing its nuclear repressive function.

160 So far, RIP140 arginine methylation links to adipocyte differentiation in a physiological 161 context. RIP140 expression enhances fat accumulation in differentiated adipocytes cells by 
162 inhibiting lipolysis enzyme expression, whereas a constitutive hypermethylated mutant

163 (where arginine is replaced by phenylalanine) failed to exert an effect on fat accumulation

164 because of reduced repressor activity [29]. Because RIP140 repressive activity is inhibited by

165 arginine methylation and because RIP140 is a key factor in estrogen signaling, it will be of 166 interest to verify whether RIP140 arginine methylation plays a role in estrogen genomic and 167 non-genomic pathways.

168

169

170

171

172

173

174

175

176

177

178

179

180

181

182

183

184

185

186

\section{Arginine methylation cross-talks with other modifications}

\section{A code for protein PTMs}

This concept has been initially proposed for histone tails which are heavily modified by methylation, in addition to other modifications like acetylation, phosphorylation, and ubiquitination. These epigenetic marks (defined as the "histone code") extend the genetic message beyond DNA sequences. They can be recognized or "read" by non-histone proteins containing for instance bromo- or chromodomains and participate in chromatin remodelling and transcriptional regulation [30, 31]. An important point deals with cross-talks that exist between the different PTMs targeting histone tails, i.e. the modification of one residue influencing the modification of neighbouring amino acids. Finally, recent publications extended these observations to non-histone proteins, with nuclear receptor and coregulator PTM coding emerging as a major level of regulation [32].

Lysine methylation of RIP140

RIP140 has been described as a substrate for several PTMs, including phosphorylation, acetylation and arginine methylation [33]. PTMs affect its subcellular distribution, protein- 
187 protein interaction, and biological activity in adipocyte differentiation. Huq et al. found recently that endogenous RIP140 is also modified by lysine methylation in differentiated 3T3L1 cells [34]. Using mass spectrometry, they found three lysine residues (K591, K653, and K757) as potential methylation sites. The loss of lysine methylation by mutation of the target

191 sites enhances arginine methylation, suggesting a communication between lysine and arginine 192 methylation. This study unraveled a potential code of modifications between lysine and 193 arginine methylation, which regulates the functionality of a non-histone protein.

\section{Phosphorylation regulates arginine methylation}

The proximity of SRC-3 methylation and phosphorylation sites suggests potential cross- talk between methylation and phosphorylation of SRC-3. Indeed, Naeem et al. showed that

198 phosphorylation of SRC-3 decreased its methylation by approximately fivefold, indicating that prior phosphorylation antagonizes methylation at least in vitro [26]. By contrast, RIP140 phosphorylation by $\mathrm{PKC} \varepsilon$ triggers its arginine methylation by inducing subsequent recruitment of the chaperone 14-3-3 necessary for PRMT1 recruitment and therefore RIP140 methylation [35]. The combination of these PTMs stimulates RIP140 nuclear export and decreases its repressive activity. Altogether, these studies show that arginine methylation of a non-histone protein can be influenced by phosphorylation, enhancing the arguments in favor of a code of modifications extended to coregulators.

\section{Regulation of PRMT activity: effects on estrogen signaling}

\section{Protein-protein interactions}

Recent studies demonstrate that the methyltransferase activity of PRMTs can be modulated by

210 protein-protein interactions. PRMT1 was initially identified as an interactor of the 211 antiproliferative proteins BTG1 (B-cell translocation gene 1) and TIS21/BTG2, stimulating its 
activity towards selected substrates $[36,37]$. PRMT1 activity is also modulated in a substrate-

213 dependent manner by the BTG protein partner, hCAF1 (CCR4 associated factor 1) [38],

214 which specifically inhibits Sam68 and histone H4 Arg3 methylation [39]. Notably histone H4,

215 when methylated by PRMT1 at Arg3, becomes a better substrate for p300, whereas

216 acetylation of $\mathrm{H} 4$ by $\mathrm{p} 300$ inhibits its methylation by PRMT1 [40, 41]. This cross-talk has

217 been described to contribute to the complex "histone code" in hormone signaling. Because

218 both hCAF1 [42] and PRMT1 [18] have been described as transcriptional regulators of the

219 nuclear receptor response, these results suggest a putative mechanism for hCAF1 in estrogen-

220 stimulated transcription through participation in PTM coding.

221 CARM1 activity is also regulated by protein-protein interactions. Indeed, CARM1 is a 222 component of a nucleosomal methylation activator complex (NUMAC) and interacts with 223 BRG1 (brahma-related gene 1), among others [43]. Once CARM1 interacts with BRG1, it can 224 then methylate histones. Moreover, CARM1 and BRG1 are both recruited to ER-target genes 225 and cooperatively activate ER-dependent transcription [43]. Therefore, modulation of PRMT1 226 and CARM1 activities by protein-protein interaction can be considered an important 227 component of regulation in estrogen signaling pathway.

\section{Phosphorylation of CARM1}

230 The methyltransferase activity of CARM1 is negatively regulated by phosphorylation. Two 231 groups describe phosphorylation of CARM1 at two different serine residues (S229, S217) 232 during mitosis $[44,45]$. Both phosphorylations abolish CARM1's ability to bind the methyl 233 donor adenosyl-methionine and subsequently inhibit CARM1 methyltransferase activity. In 234 both cases, CARM1 transactivation of estrogen receptor-dependent transcription is reduced. 235 Moreover, phosphorylation at S217 promotes CARM1 cytoplasmic localization, which occurs 236 mainly during mitosis, suggesting that the CARM1 methyltransferase activity is turned off 
during mitosis when gene transcription is silent and turned on in G1 phase when gene

238 transcription becomes active. Deregulation of this precise switch of CARM1 activity may

239 affect progression of the cell cycle in breast cancer cells. Indeed, it was shown that CARM1

240 is involved in estrogen-induced cell cycle progression of MCF-7 breast cancer cells [46].

\section{Arginine methylation and estrogen-dependent cancers}

Prmt knockout mice have been developed, providing interesting findings on the relevance of arginine methylation in vivo [1]. For instance, Prmt1 and Carml gene disruptions result in an

embryonically lethal phenotype and neonatal death respectively, confirming a fundamental role for these enzymes in cellular metabolism [47, 48]. However, until now, these genetically modified animals have not provided information regarding roles for PRMT in estrogendependent physiological processes. By contrast, recent studies using microarray and quantitative PCR-based approaches described the aberrant expression of arginine methylation enzymes in estrogen-dependent cancers (Table 2).

\section{PRMT expression in estrogen-related cancers}

254 Several recent papers analyzed PRMT expression in estrogen-dependent cancers. Prmt 1 and

255 Fbxo11 expression is up-regulated in high tumor grade breast carcinomas [49, 50] and Prmt2, 2565 and 10 expression is down-regulated in breast carcinomas [51, 52]. In ovarian 257 adenocarcinomas, Prmt1, 2 and 5 expression is down-regulated compared to normal tissues $258 \quad[53,54]$.

259 Prmt 1 isoforms exist as a result of alternative mRNA splicing, and amino acid sequence 260 comparison indicates that they are all enzymatically active, but with different $\mathrm{N}$-terminal 261 hydrophobic regions. Goulet et al. found that the expression profile of Prmt 1 splicing variants 
262 is altered in breast cancer [55]. Furthermore, this study showed that increased Prmt 1 263 expression was detected in human breast tumor samples compared with adjacent normal 264 breast tissue, confirming the studies mentioned previously. Strikingly, increased arginine265 methylated protein levels were also observed in breast cancer cell lines. Therefore, an altered 266 Prmt 1 isoform expression profile correlates with a differential pattern of arginine methylation 267 in breast cancer cell lines, suggesting that misregulation of arginine methylation could 268 contribute to the propagation of breast cancer.

Immunohistochemistry detected significant PADI4 expression in various malignancies 272 including breast carcinomas, endometrial carcinomas and uterine adenocarcinomas, with no 273 detectable PADI4 expression in benign and healthy tissues [56]. Quantitative PCR and western blot analyses also showed higher PADI4 mRNA and protein levels in malignant tissues compared to benign and non-tumor tissues [57]. Interestingly, in MCF7 breast cancer cells, PADI4 mRNA expression gradually increased with time after estradiol stimulation

277 through both classical and non-classical ER-mediated pathways [58]. Altogether, these results 278 suggest increased PADI4 expression in breast cancer tissues, probably in response to estradiol.

\section{CARM1/E2F1 breast cancer growth induced by estrogens}

282 ER $\alpha$ controls the expression of cell cycle genes which in turn mediate breast cancer 283 proliferation. Frietze et al. showed that CARM1 is essential for estrogen-induced cell cycle 284 progression in MCF-7 breast cancer cells. Upon silencing of CARM1 by siRNA, the E2285 mediated stimulation of MCF-7 cell cycle progression was strongly reduced. This silencing 286 resulted in decreased expression of E2F1 and E2F1-target genes (cyclin E1, cyclin A, 
cdc25A), providing a direct link between CARM1 and cell cycle regulation and identifying

288 CARM1 as a potential new target in the treatment of estrogen-dependent breast cancer [46].

289 Aberrant expression of CARM1 has also been linked to human breast cancer [59], with

290 elevated CARM1 levels found in aggressive breast tumors that also express high levels of the 291 oncogenic coactivator AIB1 (amplified in breast cancer 1). Compiled high levels of CARM1 292 and AIB1 could work in synergy to enhance target gene expression and thereby cell 293 proliferation.

\section{ER $\alpha$ methylation in breast cancer}

296 In MCF-7 cells, E2-induced ER $\alpha$ methylation is transitory suggesting the involvement of a not yet identified arginine demethylase whose expression or activity could be deregulated in breast cancer [20]. The evaluation of methylated ER $\alpha$ with a specific antibody showed that $\mathrm{ER} \alpha$ is hypermethylated in $50 \%$ of human breast tumors [20]. Because $\mathrm{ER} \alpha$ methylation is necessary for estrogen-induced cellular kinase pathways, this deregulation in breast cancer could lead to sustained activation of those kinases which in turn would activate estrogen 302 signaling. This bidirectional crosstalk appears to play a critical role in breast cancer development by maintaining the activation of signaling pathways and survival of breast cancer cells even in the presence of tamoxifen [60]. It is then tempting to speculate that the

305 deregulation of $\mathrm{ER} \alpha$ methylation may be involved in breast tumorigenesis and resistance to hormonal therapy. Further analyses are needed to consider methylated ER $\alpha$ a new prognostic 307 marker.

\section{Concluding remarks}

310 Arginine methylation impacts various levels of regulation in estrogen signaling, and thereby appears to be a key regulatory event in genomic and non-genomic estrogen actions. In 
genomic events, arginine methylation of transcriptional coregulators influences coregulators

313 complex formation, activity, subcellular localization, and stability. Methylation of histones

314 participates in chromatin remodeling in concert with other PTMs according to the histone

315 code. In non-genomic events, estrogen receptor methylation is necessary for the formation of

316 a transduction signaling complex and may participate in downstream events. Although, recent

317 findings strongly enhanced our knowledge of the role of arginine methylation in estrogen

318 signaling, a complete understanding will only be realized through answering fundamental

319 questions (Box 3). For instance, since expression of other PRMTs beside PRMT1 and

320 CARM1 is deregulated in estrogen-dependent cancers (Table 2), could other PRMTs be also

321 involved in genomic and non-genomic estrogen regulatory mechanisms? Are other substrates

322 implicated in estrogen signaling?

323 Deregulation of arginine methylation, methyltransferases and demethylases are described in estrogen-related cancers, strengthening the notion of a connection between arginine methylation patterns and cancer progression. Moreover, hypermethylation of ER $\alpha$ in human 326 breast cancers may indicate that methylarginine proteins represent novel interesting 327 prognostic biomarkers. Moreover, inhibiting methyltransferase activity, in particular that of PRMT1 and CARM1, by selective molecules appears as a potential therapeutic tool.

329 Recently, using an approach based on a protein virtual screen, Spannhoff et al. identified 330 specific PRMT1 inhibitors. These compounds operate as a brake on steroid hormone actions, 331 suggesting their potential for future drug development in cancer therapy [61]. There is little 332 doubt that detailed insights into the function and regulation of arginine methylation will 333 unravel the pathogenesis of various diseases, in particular hormone-dependent cancers, and 334 eventually contribute to the discovery of novel biological markers or therapeutic targets. 


\section{Box 1. Arginine methylation enzymes}

337 The enzymes responsible for arginine methylation are called protein arginine 338 methyltransferases (PRMTs). So far, eleven members have been identified in the PRMT 339 family [62, 63]. PRMT-encoding genes are well-conserved through evolution [63], sharing 340 common structural and functional domains (Figure I). Although circumstantial evidence over 341 the past 40 years depicted arginine methylation as an irreversible PTM, some data suggest 342 that this modification could be reversed. For instance, histone H4 is transiently and cyclically 343 methylated on arginine 3 [64] and estrogen receptor $\alpha$ methylation also appears transient [20].

344 While enzymes capable of removing or preventing such methylation have been identified, 345 their roles are still controversial. Peptidylarginine deiminase 4 (PADI4) has been described to 346 convert monomethylated arginine to citrulline by deimination [27, 65, 66]. However, a full 347 reversion would then need an enzyme to convert citrullines to arginine residues. More 348 importantly, PADI enzymes do not deiminate methylated arginine residues in vitro [67, 68], 349 and it seems rather that histone citrunillation simply interferes with methylation of arginine 350 residues. The first histone demethylase removing asymmetrical dimethylation at arginine 2 of 351 histone $\mathrm{H} 3$ and symmetrical dimethylation at arginine 3 of histone $\mathrm{H} 4$ is the Jumonji domain352 containing 6 protein (JMJD6) [69]. However, no publication has confirmed these results and, 353 very recently, the Bottger's group demonstrated that JMJD6 is a lysine hydroxylase involved 354 in RNA splicing indicating that demethylation of methylarginine residues is not its major 355 activity [70]. Altogether, this suggests that additional arginine demethylases remain to be 356 identified. 


\section{Box 2. Estrogen receptor and coregulator complexes}

360 Estrogens or anti-estrogens induce ER ligand binding domain (LBD) conformational change,

361 DNA binding to specific response elements in promoter regions of target genes and 362 recruitment of coregulator complexes [4]. Agonist-induced LBD conformational change 363 allows recruitment of coactivator complexes composed of p160 coactivators or PGC-1 $\alpha$ and 364 of histone-modifying enzymes (p300, CARM1 and PRMT1), whereas antagonists permit 365 recruitment of corepressors complexes containing SMRT, NCoR and HDAC enzymes. 366 Recruitment of coactivator complexes helps to pull down chromatin remodeling ATPase 367 complexes (e.g. BRG1, SWI/SNF1), which participate in chromatin remodeling. This event 368 facilitates the recruitment of the Mediator complex and thereby of the transcription machinery 369 (which contains among others the RNA Polymerase II and the TATA Binding Protein, TBP) 370 to the initiation start point [6]. Enzymes associated with coactivator complexes are 371 acetyltransferases (p300, SRC-1, SRC-3) and methyltransferases (CARM1, PRMT1). They 372 modify histone tails inducing the open state of chromatin and gene expression. On the 373 contrary, HDACs deacetylate histones leading to the closed conformation of chromatin and 374 gene repression. In the presence of estradiol, ER can also bind corepressors, such as RIP140 375 which interacts with HDACs, leading to gene repression (Figure I). 


\section{Box 3. Outstanding questions} \\ - Arginine methylome and methylarginine target proteins}

381 A fundamental issue is to define the entire arginine methylome in estrogen signaling, i.e. to

382 set up proteomic approaches with high-performance mass spectrometry methods in order to

383 describe all the methylated arginine residues in proteins involved in the estrogen pathway. In

384 addition, although several methyl lysine-binding proteins have been identified [71], effectors

385 for arginine-methylated proteins remain to be found. Actually, only three mammalian proteins

386 have been demonstrated to bind methylarginine motifs through their Tudor domains [72, 73].

387 Defining the proteins which recognize methylated arginines of estrogen receptors and 388 coregulators will certainly enhance our understanding of the downstream cascades dependent 389 on arginine methylation in estrogen signaling.

\section{0 - Regulation of arginine methylation}

391 Studies addressing the integration of signal transduction and arginine methylation pathways 392 are also needed. Specifically, how are arginine methylation and PRMT activities regulated by 393 PTMs such as phosphorylation, acetylation, ubiquitination, sumoylation and trans- or

394 automethylation? How are PRMT activity and expression regulated by estrogens or other 395 stimuli? Finally, we also clearly need a better characterization of the enzymes that 396 demethylate arginine residues.

\section{- In vivo function of arginine methylation}

398 Most of the published studies discuss the importance of arginine methylation in a cellular 399 context. It will be of great interest to elucidate further the in vivo function of the modifications 400 in estrogen target tissues. To achieve this goal, conditional knock-out approaches targeting 401 specifically breast, ovarian or uterus tissues together with knock-in strategies in mice with 402 unmethylatable mutants will be of a great help. 
This work was supported by a grant from the "Association pour la Recherche sur le Cancer"

Contre le Cancer"

\section{References}

1. Bedford, M.T., and Clarke, S.G. (2009) Protein arginine methylation in mammals: who, what, and why. Mol Cell 33, 1-13

2. Bedford, M.T., and Richard, S. (2005) Arginine methylation an emerging regulator of protein function. Mol Cell 18, 263-272 Biotechnol Annu Rev 14, 203-224

4. O'Malley, B. (2008) The Year in Basic Science: nuclear receptors and coregulators. Mol Endocrinol 22, 2751-2758

5. Lonard, D.M., and O'Malley, B.W. (2006) The expanding cosmos of nuclear receptor coactivators. Cell 125, 411-414

6. Rosenfeld, M.G. et al. (2006) Sensors and signals: a coactivator/corepressor/epigenetic code for integrating signal-dependent programs of transcriptional response. Genes Dev 20, $1405-1428$

7. Lee, D.Y. et al. (2005) Role of protein methylation in regulation of transcription. Endocr Rev 26, 147-170

8. Augereau, P. et al. (2006) The nuclear receptor transcriptional coregulator RIP140. Nucl Recept Signal 4, e024

9. Jones, P.L., and Shi, Y.B. (2003) N-CoR-HDAC corepressor complexes: roles in transcriptional regulation by nuclear hormone receptors. Curr Top Microbiol Immunol 274, 237-268

10. Bjornstrom, L., and Sjoberg, M. (2005) Mechanisms of estrogen receptor signaling: convergence of genomic and nongenomic actions on target genes. Mol Endocrinol 19, 833842

11. Migliaccio, A. et al. (2006) Crosstalk between EGFR and extranuclear steroid receptors. Ann N Y Acad Sci 1089, 194-200

12. Acconcia, F. et al. (2005) Palmitoylation-dependent estrogen receptor alpha membrane localization: regulation by 17beta-estradiol. Mol Biol Cell 16, 231-237

13. Greger, J.G. et al. (2007) Phosphorylation of MNAR promotes estrogen activation of phosphatidylinositol 3-kinase. Mol Cell Biol 27, 1904-1913

14. Azuma, K. et al. (2009) Association of estrogen receptor alpha and histone deacetylase 6 causes rapid deacetylation of tubulin in breast cancer cells. Cancer Res 69, 2935-2940 15. Litt, M. et al. (2009) Histone arginine methylations: their roles in chromatin dynamics and transcriptional regulation. Biosci Rep 29, 131-141 
16. Wysocka, J. et al. (2006) Histone arginine methylation and its dynamic regulation. Front Biosci 11, 344-355

448 17. Pal, S., and Sif, S. (2007) Interplay between chromatin remodelers and protein arginine methyltransferases. J Cell Physiol 213, 306-315

450 18. Koh, S.S. et al. (2001) Synergistic enhancement of nuclear receptor function by p160 coactivators and two coactivators with protein methyltransferase activities. J Biol Chem 276, 1089-1098

19. Chen, D. et al. (1999) Regulation of transcription by a protein methyltransferase. Science 284, 2174-2177

20. Le Romancer, M. et al. (2008) Regulation of estrogen rapid signaling through arginine methylation by PRMT1. Mol Cell 31, 212-221

21. Lahusen, T. et al. (2009) The role and regulation of the nuclear receptor co-activator AIB1 in breast cancer. Breast Cancer Res Treat 116, 225-237

22. Harigopal, M. et al. (2009) Estrogen receptor co-activator (AIB1) protein expression by automated quantitative analysis (AQUA) in a breast cancer tissue microarray and association with patient outcome. Breast Cancer Res Treat 115, 77-85

23. Torres-Arzayus, M.I. et al. (2004) High tumor incidence and activation of the PI3K/AKT pathway in transgenic mice define AIB1 as an oncogene. Cancer Cell 6, 263-274

24. Kuang, S.Q. et al. (2004) AIB1/SRC-3 deficiency affects insulin-like growth factor I signaling pathway and suppresses v-Ha-ras-induced breast cancer initiation and progression in mice. Cancer Res 64, 1875-1885

25. Feng, Q. et al. (2006) Signaling within a coactivator complex: methylation of SRC3/AIB1 is a molecular switch for complex disassembly. Mol Cell Biol 26, 7846-7857

26. Naeem, H. et al. (2007) The activity and stability of the transcriptional coactivator p/CIP/SRC-3 are regulated by CARM1-dependent methylation. Mol Cell Biol 27, 120-134

27. Lee, Y.H. et al. (2005) Regulation of coactivator complex assembly and function by protein arginine methylation and demethylimination. Proc Natl Acad Sci U S A 102, 36113616

28. Teyssier, C. et al. (2005) Activation of nuclear receptor coactivator PGC-1alpha by arginine methylation. Genes Dev 19, 1466-1473

29. Mostaqul Huq, M.D. et al. (2006) Suppression of receptor interacting protein 140 repressive activity by protein arginine methylation. Embo J 25, 5094-5104

30. Kouzarides, T. (2007) Chromatin modifications and their function. Cell 128, 693-705

31. Taverna, S.D. et al. (2007) How chromatin-binding modules interpret histone modifications: lessons from professional pocket pickers. Nat Struct Mol Biol 14, 1025-1040

32. O'Malley, B.W. et al. (2008) Cracking the coregulator codes. Curr Opin Cell Biol 20, 310-315

33. Mostaqul Huq, M.D. et al. (2008) Post-translational modifications of nuclear corepressor RIP140: a therapeutic target for metabolic diseases. Curr Med Chem 15, 386-392

34. Huq, M.D. et al. (2009) Lysine methylation of nuclear co-repressor receptor interacting protein 140. J Proteome Res 8, 1156-1167

35. Gupta, P. et al. (2008) PKCepsilon stimulated arginine methylation of RIP140 for its nuclear-cytoplasmic export in adipocyte differentiation. PLOS ONE 3, e2658

36. Berthet, C. et al. (2002) Interaction of PRMT1 with BTG/TOB proteins in cell signalling: molecular analysis and functional aspects. Genes Cells 7, 29-39

37. Lin, W.J. et al. (1996) The mammalian immediate-early TIS21 protein and the leukemia-associated BTG1 protein interact with a protein-arginine N-methyltransferase. $J$ Biol Chem 271, 15034-15044 
38. Rouault, J.P. et al. (1998) Interaction of BTG1 and p53-regulated BTG2 gene products with mCaf1, the murine homolog of a component of the yeast CCR4 transcriptional regulatory complex. J Biol Chem 273, 22563-22569

39. Robin-Lespinasse, Y. et al. (2007) hCAF1, a new regulator of PRMT1-dependent arginine methylation. J Cell Sci 120, 638-647

40. Wang, H. et al. (2001) Methylation of histone $\mathrm{H} 4$ at arginine 3 facilitating transcriptional activation by nuclear hormone receptor. Science 293, 853-857

41. Huang, S. et al. (2005) Methylation of histone H4 by arginine methyltransferase PRMT1 is essential in vivo for many subsequent histone modifications. Genes Dev 19, 18851893

42. Prevot, D. et al. (2001) Relationships of the antiproliferative proteins BTG1 and BTG2 with CAF1, the human homolog of a component of the yeast CCR4 transcriptional complex: involvement in estrogen receptor alpha signaling pathway. J Biol Chem 276, 9640-9648

43. Xu, W. et al. (2004) A methylation-mediator complex in hormone signaling. Genes Dev $18,144-156$

44. Higashimoto, K. et al. (2007) Phosphorylation-mediated inactivation of coactivatorassociated arginine methyltransferase 1. Proc Natl Acad Sci U S A 104, 12318-12323

45. Feng, Q. et al. (2009) Biochemical control of CARM1 enzymatic activity by phosphorylation. $J$ Biol Chem

46. Frietze, S. et al. (2008) CARM1 regulates estrogen-stimulated breast cancer growth through up-regulation of E2F1. Cancer Res 68, 301-306

47. Pawlak, M.R. et al. (2000) Arginine N-methyltransferase 1 is required for early postimplantation mouse development, but cells deficient in the enzyme are viable. Mol Cell Biol 20, 4859-4869

48. Yadav, N. et al. (2003) Specific protein methylation defects and gene expression perturbations in coactivator-associated arginine methyltransferase 1-deficient mice. Proc Natl Acad Sci U S A 100, 6464-6468

49. Miller, L.D. et al. (2005) An expression signature for p53 status in human breast cancer predicts mutation status, transcriptional effects, and patient survival. Proc Natl Acad Sci U S A 102, 13550-13555

50. van 't Veer, L.J. et al. (2002) Gene expression profiling predicts clinical outcome of breast cancer. Nature 415, 530-536

51. Richardson, A.L. et al. (2006) X chromosomal abnormalities in basal-like human breast cancer. Cancer Cell 9, 121-132

52. Finak, G. et al. (2008) Stromal gene expression predicts clinical outcome in breast cancer. Nat Med 14, 518-527

53. Ivshina, A.V. et al. (2006) Genetic reclassification of histologic grade delineates new clinical subtypes of breast cancer. Cancer Res 66, 10292-10301

54. Hendrix, N.D. et al. (2006) Fibroblast growth factor 9 has oncogenic activity and is a downstream target of Wnt signaling in ovarian endometrioid adenocarcinomas. Cancer Res $66,1354-1362$

55. Goulet, I. et al. (2007) Alternative splicing yields protein arginine methyltransferase 1 isoforms with distinct activity, substrate specificity, and subcellular localization. J Biol Chem 282, 33009-33021

56. Chang, X., and Han, J. (2006) Expression of peptidylarginine deiminase type 4 (PAD4) in various tumors. Mol Carcinog 45, 183-196

57. Chang, X. et al. (2009) Increased PADI4 expression in blood and tissues of patients with malignant tumors. BMC Cancer 9,40 
58. Dong, S. et al. (2007) Estrogen-enhanced peptidylarginine deiminase type IV gene (PADI4) expression in MCF-7 cells is mediated by estrogen receptor-alpha-promoted transfactors activator protein-1, nuclear factor-Y, and Sp1. Mol Endocrinol 21, 1617-1629 59. El Messaoudi, S. et al. (2006) Coactivator-associated arginine methyltransferase 1 (CARM1) is a positive regulator of the Cyclin E1 gene. Proc Natl Acad Sci U S A 103, 13351-13356

60. Tokunaga, E. et al. (2006) The association between Akt activation and resistance to hormone therapy in metastatic breast cancer. Eur J Cancer 42, 629-635

61. Spannhoff, A. et al. (2009) Cancer treatment of the future: inhibitors of histone methyltransferases. Int J Biochem Cell Biol 41, 4-11

62. Lee, Y.H., and Stallcup, M.R. (2009) Minireview: protein arginine methylation of nonhistone proteins in transcriptional regulation. Mol Endocrinol 23, 425-433

63. Krause, C.D. et al. (2007) Protein arginine methyltransferases: evolution and assessment of their pharmacological and therapeutic potential. Pharmacol Ther 113, 50-87

64. Metivier, R. et al. (2003) Estrogen receptor-alpha directs ordered, cyclical, and combinatorial recruitment of cofactors on a natural target promoter. Cell 115, 751-763

65. Cuthbert, G.L. et al. (2004) Histone deimination antagonizes arginine methylation. Cell $118,545-553$

66. Wang, Y. et al. (2004) Human PAD4 regulates histone arginine methylation levels via demethylimination. Science 306, 279-283

67. Hidaka, Y. et al. (2005) Methylation of the guanidino group of arginine residues prevents citrullination by peptidylarginine deiminase IV. FEBS Lett 579, 4088-4092

68. Raijmakers, R. et al. (2007) Methylation of arginine residues interferes with citrullination by peptidylarginine deiminases in vitro. $J$ Mol Biol 367, 1118-1129

69. Chang, B. et al. (2007) JMJD6 is a histone arginine demethylase. Science 318, 444-447

70. Webby, C.J. et al. (2009) Jmjd6 catalyses lysyl-hydroxylation of U2AF65, a protein associated with RNA splicing. Science 325, 90-93

71. Kim, J. et al. (2006) Tudor, MBT and chromo domains gauge the degree of lysine methylation. EMBO Rep 7, 397-403

72. Cote, J., and Richard, S. (2005) Tudor domains bind symmetrical dimethylated arginines. J Biol Chem 280, 28476-28483

73. Cheng, D. et al. (2007) The arginine methyltransferase CARM1 regulates the coupling of transcription and mRNA processing. Mol Cell 25, 71-83

74. Bauer, U.M. et al. (2002) Methylation at arginine 17 of histone H3 is linked to gene activation. EMBO Rep 3, 39-44

75. Saal, L.H. et al. (2007) Poor prognosis in carcinoma is associated with a gene expression signature of aberrant PTEN tumor suppressor pathway activity. Proc Natl Acad Sci U S A 104, 7564-7569

76. Cook, J.R. et al. (2006) FBXO11/PRMT9, a new protein arginine methyltransferase, symmetrically dimethylates arginine residues. Biochem Biophys Res Commun 342, 472-481

77. Troffer-Charlier, N. et al. (2007) Functional insights from structures of coactivatorassociated arginine methyltransferase 1 domains. Embo $J$ 26, 4391-4401

78. Teyssier, C. et al. (2002) Requirement for multiple domains of the protein arginine methyltransferase CARM1 in its transcriptional coactivator function. J Biol Chem 277, 46066-46072 


\section{Figures inside Boxes:}

\section{Box 1. Figure I- The PRMT family}

595 The nine members of the mammalian PRMT family are shown. Recently, two related

596 members, FBXO11 (also called PRMT9) and FBXO10, have been added to the family [63, 597 76]. The common methyltransferase domain consists of a series of short conserved motifs 598 (blue bars) that are important for binding the methyl donor and for catalysis. This catalytic 599 core region is formed by a Rossman fold and two $\alpha$ helices (black boxes). The less conserved $600 \beta$-barrel structure (gray boxes) folds against the catalytic region to form the protein substrate 601 binding cleft [77]. Additional specific motifs, such as the SH3 domain (SH3), the zinc finger $(\mathrm{ZnF})$, the myristylation motif (Myr), the FBox motif and the tetratricopeptide repeat motif (TPR) are represented in green boxes. CARM1 uniquely contains a substantial C-terminal region which contains an autonomous transcriptional activation domain [78].

605

Box 2. Figure I- Gene regulation mechanism by estrogen receptor and its coregulators

Estrogen receptor (ER) binds to specific regions called estrogen response element (ERE) represented by a pink box on the DNA target gene drawn as a thick black line wrapped around histones symbolized by yellow plots. The initiation start point is represented by a

610 black arrow. The green and red arrows represent the impacts of coactivator and corepressor complexes on chromatin state and gene transcription.

612

613 Figure 1 -Arginine methylation and estrogen signaling

614 Methylarginine proteins are involved in genomic and non-genomic estrogen actions.

615 (a) In estrogen genomic action, histones and numerous estrogen receptor $\alpha$ (ER) coregulators 616 are substrates for PRMTs. Arginine methylation regulates their transcriptional activity (PGC- 
$6171 \alpha$ ), their subcellular localization (RIP140), their stability (SRC-3) and their complex 618 assembly (p300-GRIP1). The removing methyl mark enzymatic function of PADI4 regulates 619 the assembly of the complex containing GRIP1, CARM1 and p300. This action is represented 620 by a yellow dotted line.

621 (b) In non-genomic estrogen action, induction of arginine methylation of cytoplasmic ER $\alpha$ by 622 estradiol leads to activation of downstream kinase cascades and corresponding target gene 623 activation. The role of methylated RIP140 in this non-genomic pathway remains to be 624 demonstrated. In both figures, the methyl mark is represented by a pink circle.

625

626

627 


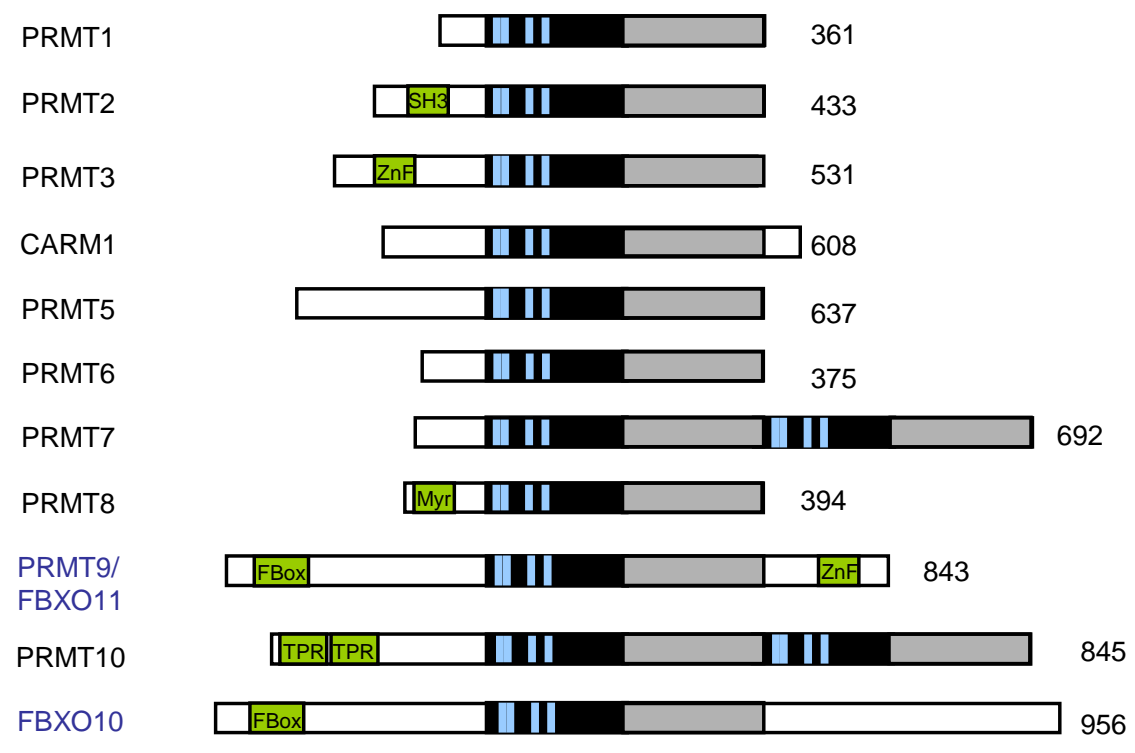

\author{
Figure I
}




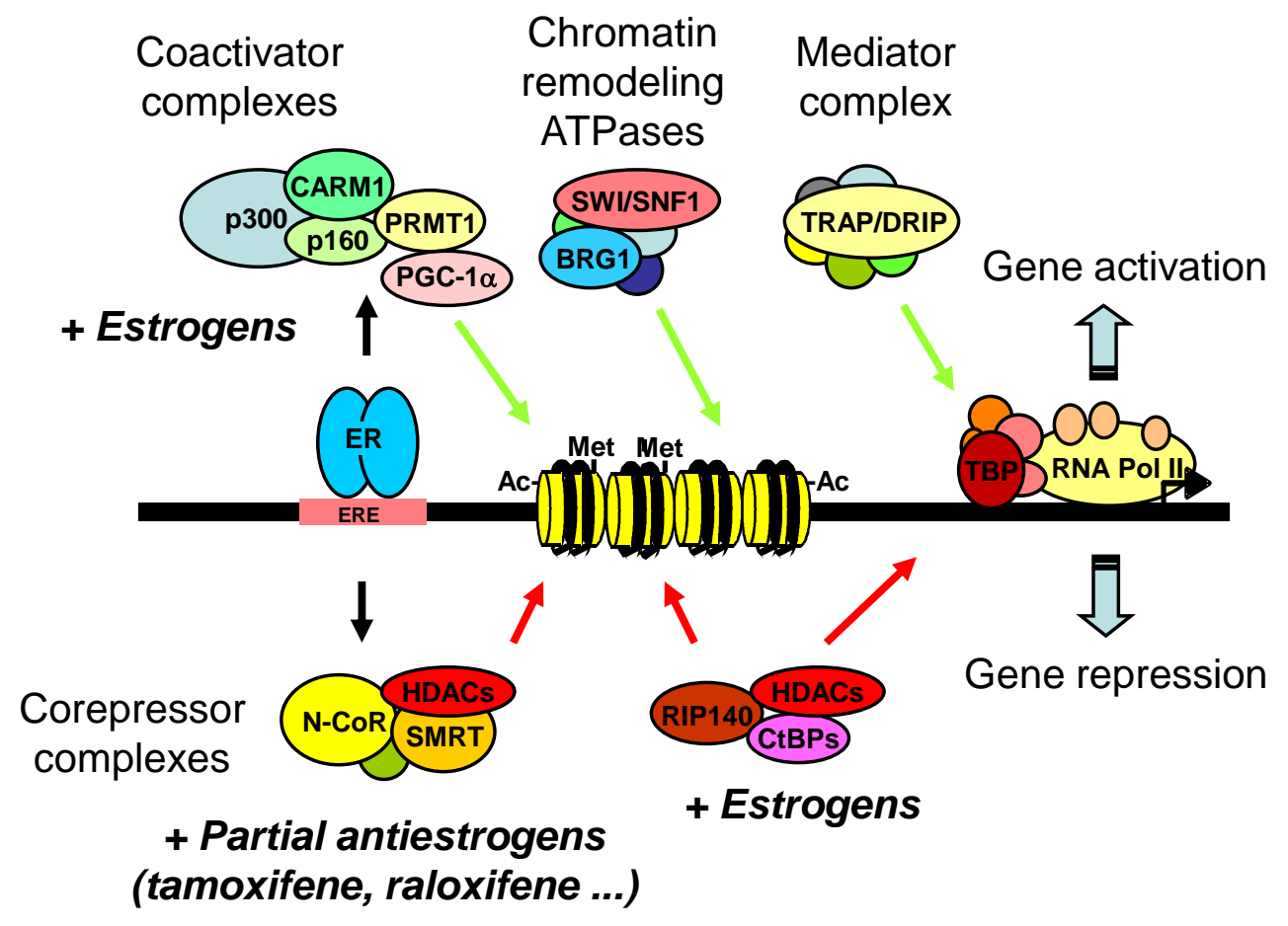

Figure I 
(a) Genomic action

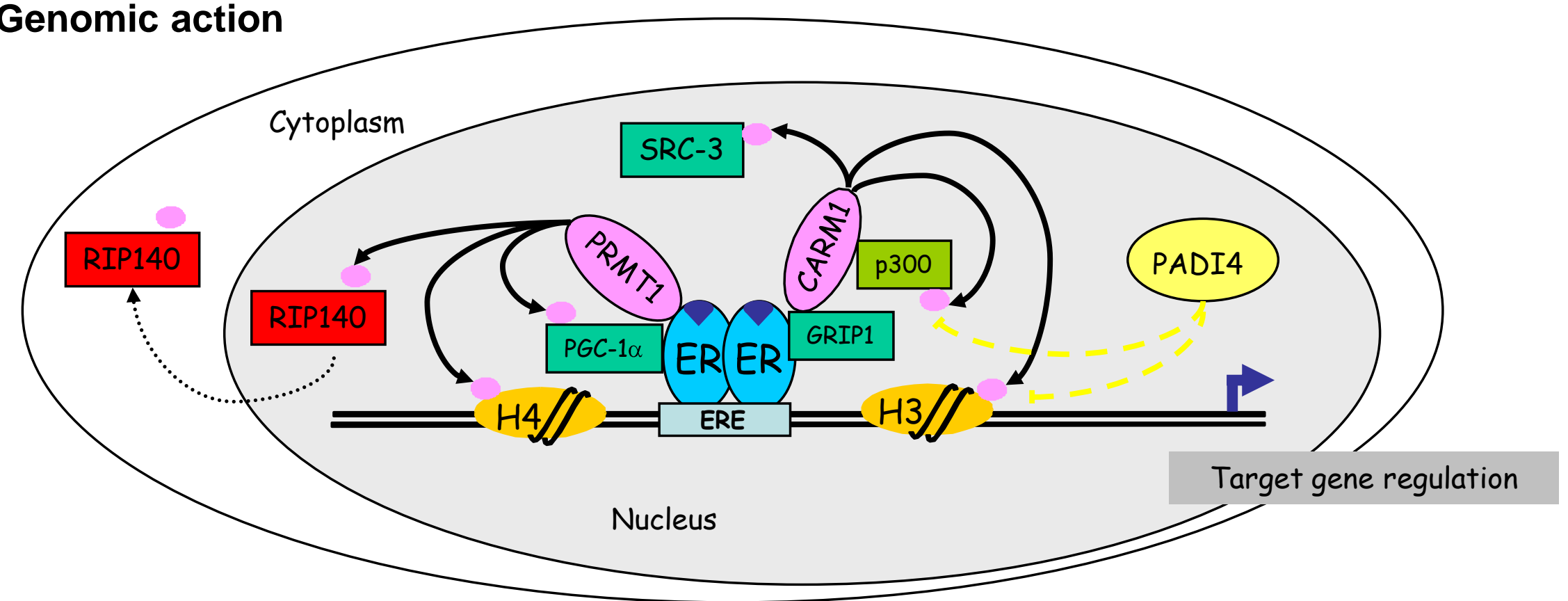

(b) Non-genomic action

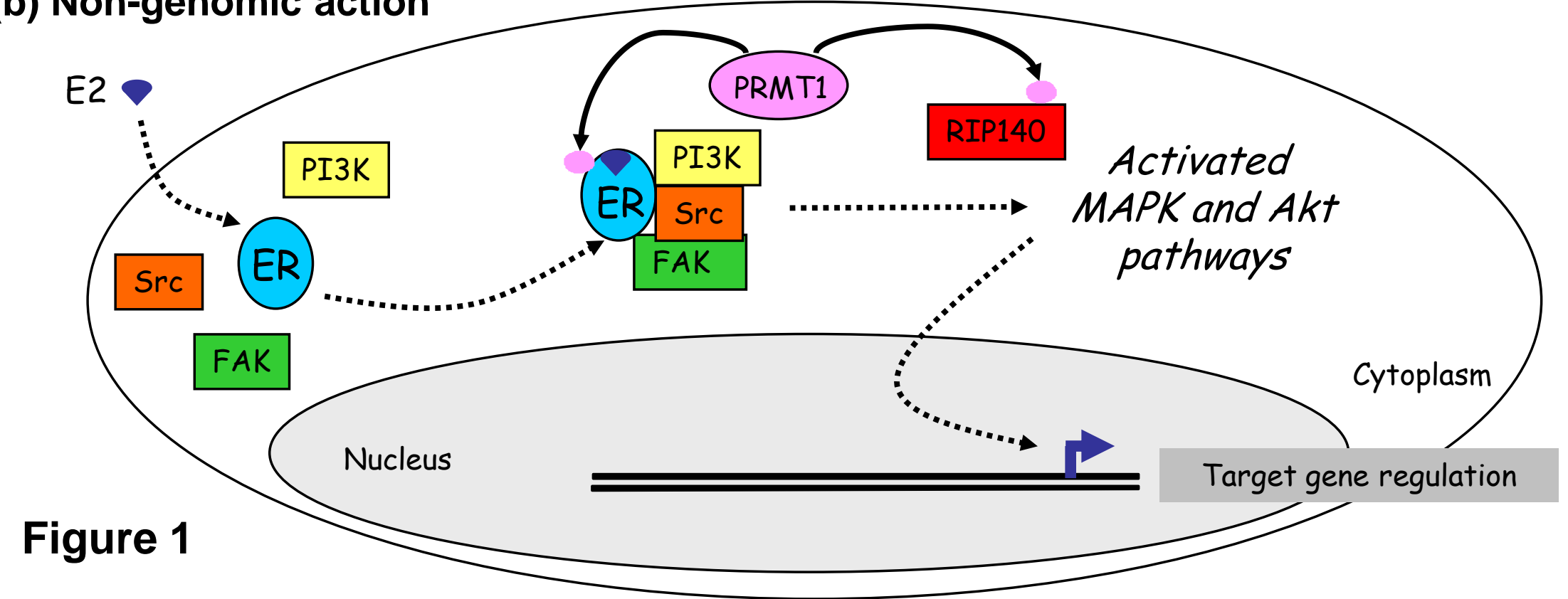


Table 1. Methylarginine proteins involved in estrogen pathway

\begin{tabular}{|lllll|}
\hline Enzyme & Substrates & Arginine (R) & Impact & Refs \\
\hline PRMT1 & Histone H4 & R3 & Gene activation & {$[40]$} \\
& ER $\alpha$ & R260 & Interaction with Src and PI3K & {$[20]$} \\
& PGC-1 $\alpha$ & R665, 667, 669 & Stimulation of coactivator activity & {$[28]$} \\
& RIP140 & R240, 650, 948 & Inhibition of repressive activity & {$[29]$} \\
CARM1 & Histone H3 & R2, 17, 26 & Gene activation & {$[19,74]$} \\
& p300/CBP & R2142 & Complex assembly regulation & {$[27]$} \\
& & R1171 & Complex assembly regulation & \\
& SRC-3 & R1178, 1184, 1195 & Activity and stability regulation & {$[25,26]$} \\
& & & & \\
\hline
\end{tabular}


Table 2. Arginine methylation enzymes in estrogen-dependent cancers

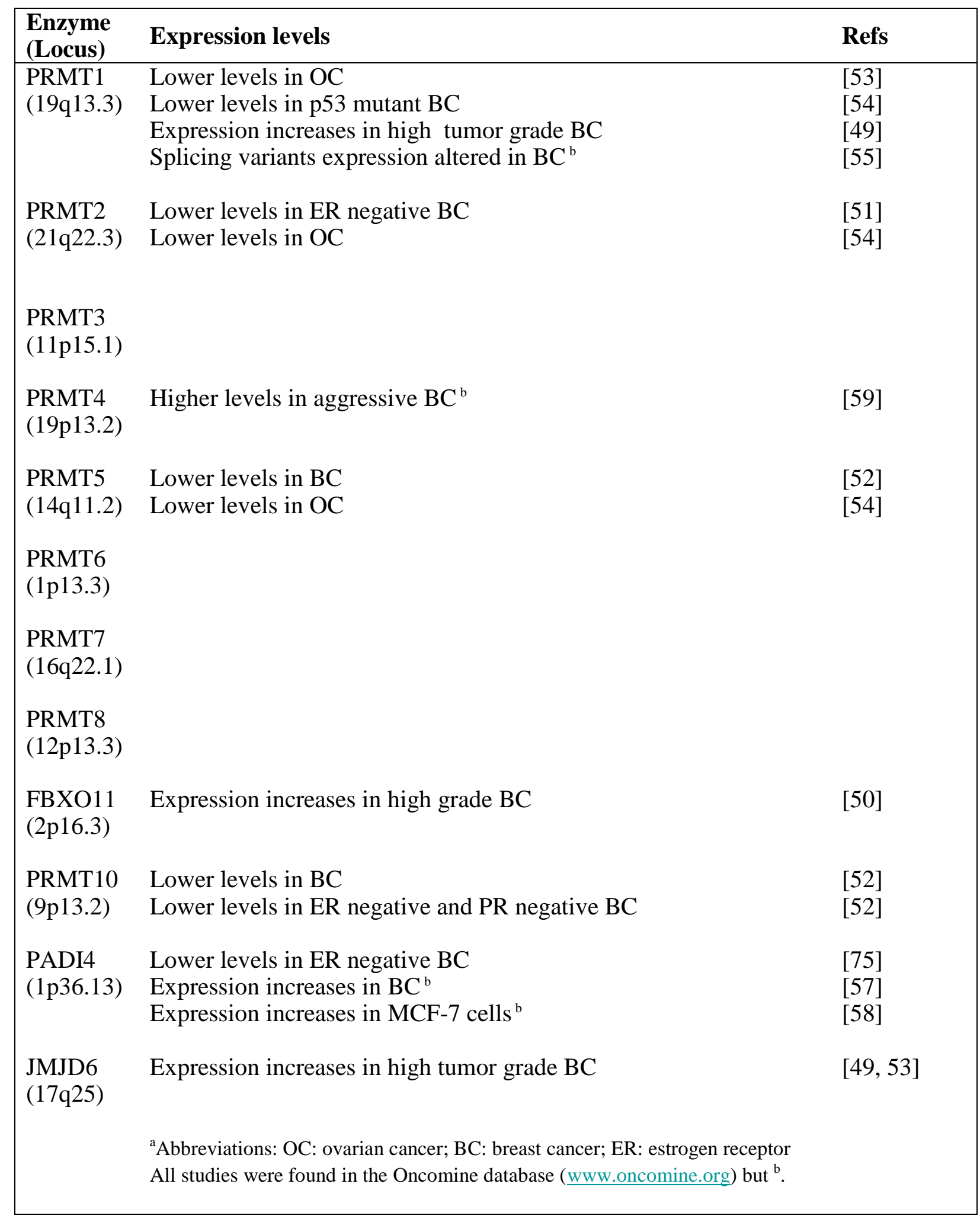

